\title{
ON A DIFFERENTIABILITY CONDTTION FOR REFLEXIVITY OF A BANACH SPACE
}

\author{
J. R. GILES
}

(Received 26 June 1969)

Communicated by J. B. Miller

In studying the geometry of normed linear space it is useful to draw attention to the following mapping.

Defintion. A mapping $x \rightarrow f_{x}$ from a normed linear space $X$ into its dual $X^{*}$ is called a support mapping if, for each $x \in S \equiv\{X \in X:\|x\|=1\}$ and real $\lambda \geqq 0$,

$$
f_{x} \in D(x) \equiv\left\{f \in S^{*}: f(x)=\|f\|=1\right\} \text { and } f_{\lambda x}=\lambda f_{x} .
$$

(The Hahn-Banach theorem guarantees that $D(x)$ is non-empty for each $x \in S$ so that such a mapping exists for every normed linear space.)

In his paper [3] the author formulated a characterisation of strong (Fréchet) differentiability of the norm of a normed linear space in terms of support mappings:

LEMMA 1. The norm of a normed linear space $X$ is strongly differentiable at $x \in S$ if and only if there exists a support mapping $x \rightarrow f_{x}$ from $X$ into $X^{*}$ which is continuous on $S$ at $x$. [3, Theorem $1($ ii)].

Such a characterisation is particularly valuable used in conjunction with the subreflexivity property of Banach spaces.

Definition. A normed linear space $X$ is said to be subreflexive if the set $P$ of continuous linear functionals which attain their norm on $S$, is dense in $X^{*}$.

E. Bishop and R. R. Phelps [1] have proved the significant result that every Banach space is subreflexive.

From Lemma 1 using the subreflexivity property the following known result can be easily deduced.

THEOREM 1. For a Banach space $X$, if the norm of $X^{*}$ is strongly differentiable on $S^{*}$ then $P=X^{*}$ and $X$ is reflexive. [3, Theorem 2].

It is the purpose of this note to deduce the following improvement of Theorem 1.

Notation. For a set $A$ in a linear space $X$ we denote by sp $(A)$ the linear span of $A$. 
THEOREM $1^{*}$. For a Banach space $X$, if the norm of $X^{*}$ is strongly differentiable on $\operatorname{sp}(P) \cap S^{*}$ then $P=X^{*}$ and $X$ is reflexive.

LemMa 2. Let $X$ be a normed linear space and $x \rightarrow f_{x}$ be a support mapping from $X$ into $X^{*}$. Consider the linear space $X$ with metric

$$
d(x, y)=\frac{1}{2}\left\{\|x-y\|+\left\|f_{x}-f_{y}\right\|\right\} .
$$

The topology of the metric $d$ is compatible with the linear structure of $X$ if and only if the support mapping is continuous on $S$.

Proof. Suppose the support mapping is continuous on $S$, then from the homogeneity property it is clear that the mapping is continuous on $X$. When $d\left(x, x_{0}\right) \rightarrow 0$ and $d\left(y, y_{0}\right) \rightarrow 0$, then $\left\|x-x_{0}\right\| \rightarrow 0$ and $\left\|y-y_{0}\right\| \rightarrow 0$ and so $\left\|(x+y)-\left(x_{0}+y_{0}\right)\right\| \rightarrow 0$. But the continuity of the support mapping implies that $\left\|f_{x+y}-f_{x_{0}+y_{0}}\right\| \rightarrow 0$, and it follows that $d\left(x+y, x_{0}+y_{0}\right) \rightarrow 0$. For a continuous support mapping it is clear that $f_{\lambda x}=\bar{\lambda} f_{x}$ for all $x \in S$ and all complex $\lambda$, and so it can be directly verified that $d\left(\lambda x, \lambda_{0} x_{0}\right) \rightarrow 0$ as $\left|\lambda-\lambda_{0}\right| \rightarrow 0$ and $d\left(x, x_{0}\right) \rightarrow 0$.

Conversely, suppose that the topology of $d$ is compatible with the linear structure. Then $d\left(x+y, x_{0}+y_{0}\right) \rightarrow 0$ as $d\left(x, x_{0}\right) \rightarrow 0$ and $d\left(y, y_{0}\right) \rightarrow 0$. For $x, y \in S$ and $\lambda$ real

$$
d(x+\lambda y, x)=\frac{1}{2}\left\{|\lambda|+\| f_{x+\lambda y}-f_{x} \mid\right\} \rightarrow 0 \quad \text { as } d(\lambda y, 0)=|\lambda| \rightarrow 0 .
$$

Therefore, $\left\|f_{x+\lambda y}-f_{x}\right\| \rightarrow 0$, and uniformly for all $y \in S$, as $|\lambda| \rightarrow 0$. But this condition is equivalent to the support mapping being continuous at $x$. [3, Lemma 1(ii)].

Lemma 3. For a Banach space $X$ where $X^{*}$ is smooth on $P \cap S^{*}$, given a support mapping $f \rightarrow F_{f}$ from $X^{*}$ into $X^{* *}$, then $P$ is complete in $X^{*}$ with respect to the metric

$$
d\left(f_{1}, f_{2}\right)=\frac{1}{2}\left\{\left\|f_{1}-f_{2}\right\|+\left\|F_{f_{1}}-F_{f_{2}}\right\|\right\} .
$$

Proof. Consider a sequence $\left\{f_{n}\right\}$ which is Cauchy in $P \cap S^{*}$ with respect to the metric $d$. Then $\left\{f_{n}\right\}$ is Cauchy in $P \cap S^{*}$ with respect to the norm of $X^{*}$ and convergentto $f \in S^{*}$ since $X^{*}$ is complete. Also $\left\{\hat{x}_{n}\right\}$, where $\hat{x}_{n}=F_{f_{n}}$ for $n=1,2, \cdots$, is Cauchy in $\hat{S}$ and so convergent to $\hat{x} \in \hat{S}$ since $X$ is complete. But

$$
\begin{aligned}
|1-f(x)| & \leqq\left|f_{n}\left(x_{n}\right)-f_{n}(x)\right|+\left|f_{n}(x)-f(x)\right| \\
& \leqq\left\|f_{n}||\right\| x_{n}-x||+\left\|f_{n}-f\right\|\|x\| .
\end{aligned}
$$

So $f(x)=1$ and $f \in P \cap S^{*}$.

These lemmas are used in establishing the result.

Proof of Theorem $1^{*}$. Since the norm of $X^{*}$ is strongly differentiable on $\operatorname{sp}(P) \cap S^{*}$, from Lemma 1 , the unique support mapping $f \rightarrow F_{f}$ from $\operatorname{sp}(P)$ into sp $(P)^{*}$ is continuous on sp $(P) \cap S^{*}$. Since $X$ is complete it is subreflexive, so 
for $f \in(\operatorname{sp}(P) \backslash P) \cap S^{*}$ there exists a sequence $\left\{f_{n}\right\} \in P \cap S^{*}$ which converges to $f$. Then $\left\{\hat{x}_{n}\right\}$, where $\hat{x}_{n}=F_{f_{n}}$, is convergent to $F_{f}$. But $\left\{\hat{x}_{n}\right\}$ is Cauchy in $\hat{S}$ so $F_{f} \in \hat{S}$, i.e. $f \in P \cap S^{*}$. Therefore $\operatorname{sp}(P)=P$.

For a support mapping $f \rightarrow F_{f}$ from $X^{*}$ into $X^{* *}, P$ is a linear space with metric

$$
d\left(f_{1}, f_{2}\right)=\frac{1}{2}\left\{\left\|f_{1}-f_{2}\right\|+\left\|F_{f_{1}}-F_{f_{2}}\right\|\right\},
$$

and since the support mapping is continuous on $P \cap S^{*}$, we have from Lemma 2 that the topology of the metric $d$ is compatible with the linear structure of $P$.

From the Metrisation theorem for linear topological spaces [4, p. 48] it follows that there exists an invariant metric on $P$ which generates the same topology as the metric $d$. Since a support mapping is norm preserving the balls centred on 0 for the metric $d$ and for the norm are equivalent. Therefore the invariant metric which generates the same topology as the metric $d$ is that induced by the norm.

But further, from Lemma 3, $P$ is complete with respect to the metric $d$. It then follows from a result of V. L. Klee $[7$, p. 84] that $P$ is complete as a normed linear space, and so $P$ is a closed subspace of $X^{*}$. However, $P$ is dense in $X^{*}$. Therefore $P=X^{*}$.

The result then follows as in Theorem 1 .

It should be noted that

1. a Banach space $X$ whose norm is strongly differentiable on $S$, is not necessarily reflexive, and

2. a Banach space $Y$ where the norm of $Y^{*}$ is strongly differentiable on $P \cap S^{*}$, is not necessarily reflexive.

The following example constructed by R. R. Phelps [6, p. 447] illustrates both these points.

Let $Y$ be the linear space $l_{1}$ of sequences $y=\left\{y_{n}\right\}$ where $\sum_{n}\left|y_{n}\right|<\infty$, with the norm

$$
\|y\|=\left\{\left(\sum_{n}\left|y_{n}\right|\right)^{2}+\sum_{n}\left(\frac{y_{n}}{2^{n}}\right)^{2}\right\}^{\frac{x}{2}}
$$

and $X$ be the linear space $c_{0}$ of sequences $x=\left\{x_{n}\right\}$ which converge to zero, with the norm

$$
\|x\|=\sup \left\{\sum_{n} x_{n} y_{n}: y=\left\{y_{n}\right\} \in Y \text { and }\|y\| \leqq 1\right\} .
$$

Phelps has shown that $X$ is a non-reflexive Banach space, $Y=X^{*}$ and the norm of $Y$ is locally uniformly rotund on $S$. He deduced from a theorem of A. R. Lovaglia [5, p. 232] that the norm of $X$ is strongly differentiable on $S$. But also, from results of D. F. Cudia [2, p. 308 and p. 296] we can deduce that the norm of $Y^{*}$ is strongly differentiable on $P \cap S^{*}$. 


\section{References}

[1] E. Bishop and R. R. Phelps, 'A proof that every Banach space is subreflexive', Bull. Amer. Math. Soc. 67 (1961), 97-98.

[2] D. F. Cudia, 'The geometry of Banach spaces. Smoothness', Trans. Amer. Math. Soc. 110 (1964), 284-314.

[3] J. R. Giles, 'On a characterisation of differentiability of the norm of a normed linear space', J. Aust. Math. Soc. 12 (1971), 106-114.

[4] J. L. Kelley and I. Namioka, 'Linear Topological Spaces' (Van Nostrand, Princeton, 1963).

[5] A. R. Lovaglia, 'Locally uniformly convex Banach spaces', Trans. Amer. Math. Soc. 78 (1955), 225-238.

[6] R. R. Phelps, 'Subreflexive normed linear spaces', Arch. Math. 8 (1957), 444-450.

[7] A. Wilansky, 'Functional Analysis' (Blaisdell, Waltham, Mass., 1964).

The University of Newcastle, N.S.W. 